\title{
Calceolaria lagunae-blancae Kraenzl., nuevo registro para la flora de Chile
}

\section{Calceolaria lagunae-blancae Kraenzl. new record for the Chilean flora}

\author{
JonAthan URRUtia* \& Pablo Bravo-Monasterio \\ Facultad de Ciencias Forestales, Universidad de Concepción, Laboratorio de Invasiones Biológicas (LIB), Casilla 160-C, \\ Concepción, Chile. \\ Instituto de Ecología y Biodiversidad (IEB), Santiago, Chile. \\ *jurrutiaestrada@gmail.com
}

\begin{abstract}
For first time in Chile is documented the presence of Calceolaria lagunae-blancae Kraenzl., a species registered only in Argentina up to now. The collection was carried out in the Aisen Region, specifically in Coyhaique Alto town. We provide a description of the species and their habitat.
\end{abstract}

Calceolaria es un género andino con más de 300 especies que se distribuye desde México hasta Tierra del Fuego (Molau 1988). Hasta hace un tiempo se incluía dentro de la familia Scrophulariaceae, sin embargo, se demostró para este grupo la inexistencia de un ancestro común (polifilético), por lo que se elevó de categoría taxonómica (Olmstead et al. 2001); y forma desde entonces junto al género Jovellana la familia Calceolariaceae. En Chile existen 51 especies de Calceolaria (Ehrhart 2000), una de las cuales fue descrita recientemente con el nombre de $C$. philippii (Eyzaguirre 2014).

Durante relevamientos florísticos realizados los años 2011 y 2013 en la Región de Aisén, se registró la presencia de una especie del género Calceolaria desconocida hasta el momento. Dicho taxa fue avistado en primera instancia por Urrutia en enero de 2011 en la localidad de Coyhaique Alto (45³0'10,45'S - 71'42'26,88'O) a 750 msnm, posteriormente en noviembre de 2013 Bravo también la registra y además la recolecta en el mismo sector. Estudios posteriores realizados en los especímenes recolectados revelaron que la identidad de dicha planta correspondía a Calceolaria lagunae-blancae Kraenzl. (Fig. 1), especie que se distribuye principalmente en la estepa argentina (Rossow 1999).

En el catálogo de Marticorena \& Quezada (1985) y en la monografía del género realizada por Ehrhart (2000) no se registra la presencia de $C$. lagunae-blancae para el territorio chileno. Las características de hábitat donde se desarrollan sus poblaciones corresponden a la ecorregión fría de estepa patagónica (SAG 2008), donde la vegetación es dominada por Festuca pallescens (St.-Yves) Parodi y Mulinum spinosum (Cav.) Pers. (Luebert \& Pliscoff 2006). Hasta el momento esta especie se considera endémica de Argentina y ocupa las provincias de Neuquén, Río Negro, Chubut y Santa Cruz (Zuloaga et al. 2008). Rossow (1999) señala que es relativamente común en dicha extensión y que prefiere ocupar ambientes de suelos pedregosos y orillas de camino. Esta última descripción es coincidente con el lugar en donde fue hallada en Chile.

DESCRIPCIÓN DE LA ESPECIE

Planta de $\pm 15 \mathrm{~cm}$ de alto, hojas en roseta, rizomatosa. Raíces delgadas. Hojas de 2 a 4,5 cm de largo por 0,6 a $1 \mathrm{~cm}$ de ancho, elípticas, enteras a dentadas, cortamente pseudopecioladas, glanduloso-pubescentes. Escapos áfilos, ebracteados, terminando en inflorescencias 3-5-floras. Cáliz pubescente, sépalos ligeramente desiguales, dos opuestos de 5 x 3,5 mm y dos de 5,5 x $4 \mathrm{~mm}$, ovados, pubescentes exteriormente y con pelos largos hacia el ápice y borde. Corola con el labio superior de $2-3 \mathrm{~mm}$, profundamente cuculado; labio inferior de 8-10 mm, con líneas horizontales internas color marrón, elipsoide a subcuadrado, glabro en la cara adaxial y glanduloso en la abaxial, elaióforo papiloso. Filamentos estaminales de $\pm 1,5 \mathrm{~mm}$, anteras de $\pm 2 \mathrm{~mm}$, elipsoides. Cápsula de $\pm 6 \mathrm{~mm}$, piriforme, glandulosa. Semillas de $\pm 1 \mathrm{~mm}$, longitudinalmente marcadas $\mathrm{y}$ ampolladas, pardo-rojizas.

\section{Material estudiado}

CHILE: Región de Aisén, Provincia de Coyhaique, Sector de Coyhaique Alto, a $35 \mathrm{~km}$ de la ciudad de Coyhaique. 750 m s.n.m. (45³0'10,45'S -71'42’26,88’O). 27-XI-2013, Pablo Bravo Monasterio (CONC 180421). 


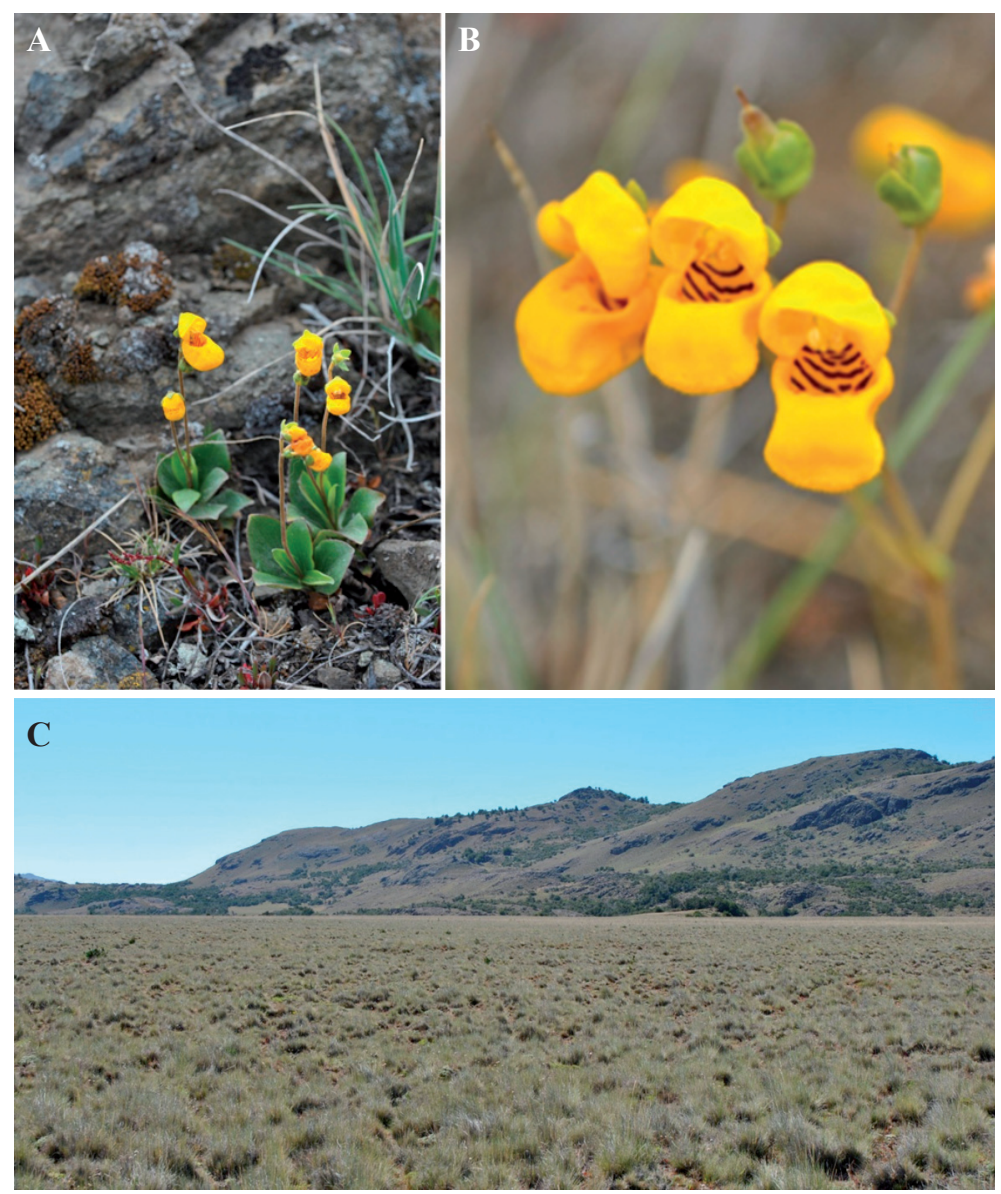

Figura 1. Calceolaria lagunae-blancae. A: individuo, B: flor, C: hábitat.

Figure 1. Calceolaria lagunae-blancae. A: individual, B: flower, C: habitat.

\section{AGRADECIMIENTOS}

Los autores agradecen a Alicia Sérsic de la Universidad Nacional de Córdoba por su ayuda en la determinación de la especie, a los proyectos FONDECYT 1100792, 1120171, ICM 05-002 y PFB-23. Este estudio se enmarca en el trabajo del Laboratorio de Invasiones Biológicas (LIB), www.lib. udec.cl.

\section{BIBLIOGRAFÍA}

Ehrhart, C. 2000. Die Gattung Calceolaria (Scrophulariaceae) in Chile. Biblioteca Botánica. 283 pp.

Eyzaguirre, M.T. 2014. Novedades florísticas en la Reserva Nacional Roblería del Cobre de Loncha: Calceolaria philippii sp. nov. (Calceolariaceae) para la flora de Chile y ampliación de la distribución de Anemone rigida Gay (Ranunculaceae). Gayana Botánica 71: 280-283.

Luebert, F. \& P. Pliscoff. 2006. Sinopsis bioclimática y vegetacional de Chile. Editorial Universitaria, Santiago. $316 \mathrm{pp}$.

Marticorena, C. \& M. Quezada. 1985. Catálogo de la flora vascular de Chile. Gayana Botánica 42: 5-157.

Molau, U. 1988. Scrophulariaceae. Part I. Calceolarieae. Flora Neotropica Monographs 47: 1-326.

Olmstead, R., C. dePamphilis, A. Wolfe, N. Young, W. Elisons \& P. ReEves. 2001. Desintegration of the Scrophulariaceae. American Journal of Botany 88: 348-361.

Rossow, R. 1999. Scrophulariaceae. En: M. Correa (ed.), Flora Patagónica Parte IV, Dicotyledones Gamopétalas (Ericaceae a Calyceraceae), pp 310-331. Colección Científica del INTA, Buenos Aires.

SAG. 2008. Guías de Condición para los Pastizales de la Ecorregión Esteparia Fría de Aysén. Gobierno Regional de Aysén. Ministerio de Agricultura Servicio Agrícola y Ganadero. 94 pp.

Zuloaga, F., O. Morrone \& M. Belgrano (eds.). 2008. Catálogo de las plantas vasculares del cono sur (Argentina, sur de Brasil, Chile, Paraguay y Uruguay). Missouri Botanical Garden Press. Saint Louis. 3348 pp.

Recibido: 04.05.15

Aceptado: 22.09.15 